\title{
Revisión de la poliposis gástrica: a propósito de un caso operado por laparoscopia, con protocolo fast track
}

\author{
Gustavo Pérez B. ${ }^{1}$, Octavio del Real V. ${ }^{1}$ y Nasser Eluzen G. ${ }^{1}$
}

'Departamento de Cirugía

Digestiva, Facultad de

Medicina, Pontificia Universidad Católica de Chile, Santiago, Chile.

Recibido el 23 de noviembre de 2016 y aceptado para publicación el 2 de febrero de 2017.

Correspondencia a: Dr. Gustavo Pérez B. gperez@med.puc.cl
Review of gastric polyposis: About a case operated by laparoscopy, with fast track protocol

Introduction: Gastric polyps are commonly found incidentally on upper gastrointestinal endoscopy. Objective: Review the most common gastric polyps and present a case of symptomatic polyps managed with laparoscopic total gastrectomy following the fast track protocol. Clinical case: Patient with symptomatic diffuse gastric polyps who had a total laparoscopic gastrectomy following the fast track protocol, who is discharged at home at fourth day post-surgery. Discussion: The most common are hyperplastic polyps, fundic gland polyps, adenomas and inflammatory fibroid polyps. Is very important to obtain biopsies to assess the histology and malignant potential. In certain cases a total gastrectomy is recommended. The fast track rehabilitation protocol is a safe method to decrease hospital stay and improve recovery.

Key words: gastric polyposis; gastrectomy; laparoscopic surgery.

\section{Resumen}

Introducción: Los pólipos gástricos suelen ser hallazgos en la endoscopia digestiva alta. Objetivo: Realizar una revisión de los pólipos gástricos más frecuentes y presentar un caso de manejo de estos con gastrectomía total laparoscópica según el protocolo fast track. Caso clínico: Se presenta el caso de un paciente hombre de 46 años, sin antecedentes mórbidos con poliposis difusa del estómago sintomática, en el que se realiza una gastrectomía total laparoscópica siguiendo el protocolo fast track, siendo dado de alta al cuarto día posoperatorio. Discusión: Los pólipos gástricos más frecuentes son los hiperplásicos, de glándula fúndica, adenomas e inflamatorios. Es fundamental obtener biopsias de estos ya que su histología comandará su manejo y pronóstico. En algunos casos, la gastrectomía total es la indicación debido a su riesgo de malignidad o sintomatología. El protocolo fast track ha demostrado ser una modalidad de manejo posoperatorio que disminuye el tiempo de estadía hospitalaria y presenta una recuperación precoz en comparación con la práctica habitual, sin comprometer la seguridad del paciente.

Palabras clave: pólipos gástricos; gastrectomía; cirugía laparoscópica.

\section{Introducción}

Los pólipos gástricos suelen ser un hallazgo en la endoscopia digestiva alta (EDA), ya que generan síntomas con baja frecuencia (hemorragia, dolor abdominal, síndrome pilórico) que hagan sospecharlos previamente a este examen ${ }^{1}$. Sin embargo, es importante reconocerlos y conocer su manejo clínico dado que algunos pólipos son potencialmente malignos. Se presenta un caso de poliposis gástrica difusa sintomática en el cual se realizó una gastrectomía total laparoscópica siguiendo un protocolo de recuperación acelerada (fast track).

\section{Caso clínico}

Hombre de 46 años, sin antecedentes mórbidos, que consulta por cuadro de 2 meses de compromiso del estado general y fatigabilidad. Se realiza estudio detectándose anemia severa (hematocrito: 
$18 \%$; hemoglobina: $4 \mathrm{~g} / \mathrm{dl}$ ), por lo que se transfunden 4 unidades de glóbulos rojos, hierro i.v. y esomeprazol $40 \mathrm{mg} / 12 \mathrm{~h}$. Se realiza colonoscopia en otro centro que informa un pólipo de sigmoides resecado durante la misma, del cual no hay biopsia disponible. Posteriormente se realiza EDA evidenciándose extensa poliposis gástrica difusa, pólipos de aspecto adenomatoso que sangran fácilmente (Figura 1). Se toman biopsias de pólipos que informan mucosa de tipo gástrico corporal de arquitectura general conservada, con signos de una inflamación crónica inactiva, superficial, leve e inespecífica, asociada a focos de edema y algunas áreas con hiperplasia glandular foveolar polipoidea. Helicobacter pylori resulta negativo. Además, se toman muestras de mucosa gástrica sana y duodenal, ambas normales. El TC de abdomen muestra poliposis gástrica difusa y esplenomegalia. Se discute el caso en equipo, y se indica gastrectomía total laparoscópica.

Se realiza el acceso laparoscópico con 4 trocares, 3 de $12 \mathrm{~mm}$, supraumbilical, línea media clavicular izquierda y en hipocondrio derecho, además de un trocar de $5 \mathrm{~mm}$ subxifoideo. Se evidencia estómago dilatado, de paredes gruesas, difícil de manipular debido a su contenido. Esqueletización de curvatura mayor y menor con Ligasure, clips a arteria gástrica derecha, sección de duodeno con engrapadora lineal cortante de $45 \mathrm{~mm}$ carga azul. Se confecciona Y de Roux cortando yeyuno aproximadamente a 30 $\mathrm{cm}$ del ángulo de Treitz y anastomosando en forma laterolateral con engrapadora lineal cortante blanca con asa alimentaria de aproximadamente $60 \mathrm{~cm}$. Se realiza sección de esófago distal con bisturí armónico; anastomosis esofagoyeyunal terminolateral, antecólica, con Vicryl 3/0 corrido más refuerzos. Se extraen piezas por incisión de Pfannenstiel de $6 \mathrm{~cm}$. Se cierra brecha mesentérica y Peterson con Seda $2 / 0$. Se instala drenaje Jackson Pratt en relación con muñón duodenal y otro en esófago-yeyuno anastomosis.

Al segundo día posoperatorio se realiza un estudio de radiografía digestiva alta con contraste oral, que no muestra filtración anastomótica, por lo que se inicia la realimentación progresiva, la cual es bien tolerada. Se retiran los drenajes y se indica el alta al cuarto día posoperatorio. Se controla el paciente a los 10 días, evidenciándose en buenas condiciones, tolerando bien el régimen líquido. La biopsia de estómago informa poliposis hiperplásica difusa que compromete difusamente el antro y cuerpo gástrico, con hiperplasia glandular, foveolar y dilatación de glándulas fúndicas, sin focos de displasia ni carcinoma, con $H$. pylori negativo.

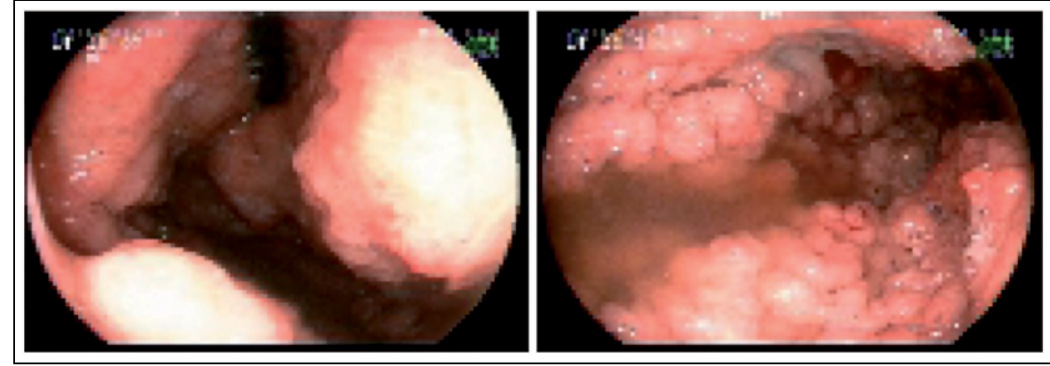

Figura 1. Endoscopia digestiva alta que muestra extensa poliposis difusa del estómago.

\section{Discusión}

Los pólipos gástricos suelen ser un hallazgo en la EDA, siendo poco frecuente que se presenten con síntomas como en este caso. Se identifican más frecuentemente 3 tipos de pólipos gástricos benignos: hiperplásicos, de glándula fúndica (PGF) e inflamatorios, y los pólipos neoplásicos: adenomas, carcinoides, tumores del estroma gastrointestinal (GIST) y adenocarcinomas. Es esencial poder obtener biopsias de estos dado que su histología comandará el manejo y pronóstico.

Los pólipos gástricos tienen una incidencia que va desde el 0,6 al 6,35\% de las EDA. La histología es variable, dependiendo de la región y del año. En Brasil, en el 2007, un 71,3\% fueron hiperplásicos, un $16,3 \%$ PGF y un $12,4 \%$ adenomatosos ${ }^{1}$; en cambio, en Estados Unidos, en el 2008, un 77\% fueron PGF, un $17 \%$ hiperplásicos, un $0,69 \%$ adenomas y un $0,1 \%$ inflamatorios, con un $2 \%$ de malignidad $^{2}$. En China, una revisión de 24.121 casos realizada por Hailong et al. comparó la distribución de pólipos gástricos entre los años 2000 y 2010, con una incidencia en las EDA de un 1\%, en la cual se evidencia una disminución significativa de los pólipos hiperplásicos (48,5 a 20,8\%), adenomas (10,3 a $3,3 \%)$, inflamatorios $(25$ a $6 \%$ ) y carcinomas $(4,4$ a $1,6 \%$ ), asociada con un aumento de los PGF $(8,8$ a $66,1 \%$ ) en ese período, explicado principalmente por la disminución de la prevalencia de H. pylori ${ }^{3}$.

Frente al hallazgo de un pólipo gástrico solitario se debe tomar una biopsia o realizar la polipectomía. Si el pólipo es mayor de $5 \mathrm{~mm}$ se recomienda polipectomía. En el caso de múltiples pólipos se recomienda polipectomía del más grande y muestras de biopsia de los otros pólipos ${ }^{4}$. Además, se deben tomar muestras de la mucosa circundante al pólipo y de los diferentes sectores gástricos para diagnosticar H. pylori y descartar la presencia de displasia o neoplasia 5 . 
Los pólipos hiperplásicos se aprecian en la EDA como pólipos suaves y brillantes, pueden tener erosiones o ser umbilicados y al microscopio se observa epitelio foveolar edematoso, distorsionado, elongado y dilatado 6 . Se asocian a inflamaciones crónicas de la mucosa gástrica, tales como la presencia de $H$. pylori ${ }^{7}$ o de gastritis atrófica autoinmune, los cuales son factores de riesgo para desarrollar cáncer gástrico ${ }^{8}$. Existe un riesgo de desarrollo de adenocarcinoma en un pólipo hiperplásico de un $0-8 \%$, el cual puede aumentar en presencia de múltiples pólipos $^{6}$. Todos los pólipos mayores de $5 \mathrm{~mm}$ deben ser resecados ${ }^{4}$. Dado que la erradicación de $H$. pylori produce regresión de los pólipos hiperplásicos, esta debe ser tratada si es objetivada ${ }^{6,7}$. Se recomienda una EDA de control en un año ${ }^{4}$.

Los PGF se encuentran generalmente en el cuerpo y fondo gástricos, son lesiones múltiples sésiles de 2-3 $\mathrm{mm}^{6}$. Se manifiestan en pacientes sin H. pylori, usuarios de inhibidores de la bomba de protones (IBP) por más de un año ${ }^{9}$ y en personas con poliposis adenomatosa familiar $(\mathrm{PAF})^{10}$. En el primer caso el riesgo de displasia y neoplasia es casi nulo ${ }^{4,9}$; en los pacientes con PAF el riesgo de displasia es del $42 \%$, pero este disminuye con el uso de $\mathrm{IBP}^{10}$, sin embargo, hay descritos casos aislados de adenocarcinoma gástrico en personas con PAF ${ }^{11}$.

Los pólipos inflamatorios son tumores del mesénquima que se originan de la mucosa y submucosa, que se visualizan como pólipos firmes erosionados y microscópicamente como depósitos de fibroblastos circunferenciales a los vasos, con infiltrado eosinófilo. No tienen potencial maligno y no presentan recurrencias una vez resecados ${ }^{6}$.

Los pólipos adenomatosos o adenomas gástricos son neoplasias de la mucosa, pueden ser planos o sésiles, en general, solitarios y erosionados. Histológicamente se caracterizan por un epitelio columnar pseudoestratificado, con núcleos atípicos elongados y con aumento de la actividad mitótica ${ }^{6}$. $\mathrm{Su}$ potencial maligno depende principalmente del grado de displasia presente: un estudio holandés que realizó seguimiento a pacientes con displasias de bajo y alto grado diagnosticó cáncer gástrico (1,5 y 10 años) a un 2,1, 3,1 y 3,9\% de los pacientes con displasia de bajo grado, y a un 24,9, 29,5 y $32,7 \%$ de quienes tenían displasia de alto grado $^{12}$. Además, existe un riesgo de un $8-59 \%$ de presentar un cáncer gástrico sincrónico ${ }^{13}$. Dado el alto riesgo de malignidad, todos los pólipos adenomatosos deben ser resecados y además se debe examinar y tomar muestras del resto del estómago para descartar la presencia de cáncer gástrico concomitante. Si no es posible realizar la polipectomía endoscópica, se debe derivar al cirujano digestivo. Se sugiere un seguimiento con EDA al año, y si no hay displasia o recurrencia, se pueden realizar EDA cada 3-5 años. Si hay displasia o se detecta cáncer, el manejo debe ser según la indicación del cirujano ${ }^{4}$.

Respecto a carcinoides, GIST y adenocarcinomas tienen su diagnóstico y manejo específico que no detallaremos en este documento.

El protocolo fast track para gastrectomías laparoscópicas ya ha sido descrito: realimentación precoz con una radiografía digestiva alta con contraste oral que certifique ausencia de filtración de la anastomosis esofagoyeyunal, sin drenajes ni sonda nasogástrica, sin anestesia peridural, uso de sonda Foley por menos de $24 \mathrm{~h}$ y alta planificada luego de las $72 \mathrm{~h}$ poscirugía. Ha tenido un buen éxito en los lugares donde se ha implementado, considerando que debe ser realizada por un cirujano experimentado, en una cirugía electiva en un paciente compensado de sus patologías crónicas, con la cooperación del anestesista, de los equipos de enfermería, nutrición y rehabilitación, y con un paciente comprometido que entienda los beneficios y riesgos de este protocolo. Los beneficios son una recuperación más rápida, menor estadía hospitalaria, recuperación laboral precoz y satisfacción del paciente, sin comprometer su seguridad ${ }^{14-16}$.

En este caso descrito los pólipos se manifestaron clínicamente por anemia crónica, lo cual es poco frecuente ya que habitualmente son un hallazgo en las EDA. Luego de la estabilización del paciente se realiza la EDA, la cual muestra múltiples pólipos de aspecto adenomatoso, pero que la biopsia informa posteriormente como pólipos hiperplásicos. Dada la presencia de múltiples pólipos, su potencial de malignidad y la anemia, se indica la gastrectomía total, la cual se decide hacer por vía laparoscópica por la ventaja en cuanto a la menor pérdida de sangre y menor estadía hospitalaria en comparación con la gastrectomía abierta ${ }^{17}$, siguiendo un protocolo fast track, excepto en cuanto a la instalación de los drenajes. Dada la buena recuperación del paciente, se realiza la radiografía digestiva alta con contraste oral al segundo día posoperatorio, la cual realizamos habitualmente para asegurar la ausencia de filtración de la anastomosis previamente a la realimentación, siendo negativa para esta. Se procede a la administración de agua vía oral, progresando a hídrico amplio bien tolerado. Dada la buena condición del paciente se indica el retiro de los drenajes y el alta al cuarto día posoperatorio. 


\section{Comentarios}

Los pólipos gástricos suelen ser un hallazgo en la EDA, dado que con baja frecuencia se manifiestan con síntomas. Es fundamental obtener biopsias de estos ya que su histología definirá su manejo y pronóstico. La mayoría de los pólipos gástricos son benignos, a pesar de lo cual en algunos casos la gastrectomía total es la indicación debido a su riesgo de malignidad o sintomatología. La gastrectomía total laparoscópica fast track es una alternativa para estos pacientes, con una rápida recuperación, realimentación y alta precoz sin aumentar los riesgos.

\section{Responsabilidades éticas}

Protección de personas y animales. Los autores declaran que para esta investigación no se han realizado experimentos en seres humanos ni en animales.
Confidencialidad de los datos. Los autores declaran que han seguido los protocolos de su centro de trabajo sobre la publicación de datos de pacientes.

Derecho a la privacidad y consentimiento informado. Los autores han obtenido el consentimiento informado del paciente $\mathrm{y} / \mathrm{o}$ sujeto referido en el artículo. Este documento obra en poder del autor de correspondencia.

\section{Financiación}

Los autores declaran no haber recibido ninguna financiación para la realización de este trabajo.

\section{Conflicto de intereses}

Los autores declaran no tener ningún conflicto de intereses.

\section{Bibliografía}

1. Morais DJ, Yamanaka A, Zeitune JM, Andreollo NA. Gastric polyps: A retrospective analysis of 26.000 digestive endoscopies. Arq Gastroenterol. 2007;44:14-7.

2. Carmack SW, Genta RM, Schuler CM, Saboorian MH. The current spectrum of gastric polyps: A 1 year national study of over 120.000 patients. Am J Gastroenterol. 2009;104:1524-32.

3. Hailong C, Bangmao W, Zhihua Z, Hui Z, Rui Q. Distribution trends of gastric polyps: An endoscopy database analysis of 24.121 northern Chinese patients. J Gastroenterol Hepatol. 2012;27:1175-80.

4. Hirota WK, Zuckerman MJ, Adler DG, Davila RE, Egan J, Leigh- ton JA, et al. ASGE guideline: The role of endoscopy in the surveillance of premalignant conditions of the upper GI tract. Gastrointest Endosc. 2006;63:570-80.

5. Sharaf RN, Shergill AK, Odze RD, Krinsky ML, Fukami N, Jain R, et al., ASGE Standards of Practice Committee. Endoscopic mucosal tissue sampling. Gastrointest Endosc. 2013;78:216-24.

6. Oberhuber G, Stolte M. Gastric polyps: An update of their pathology and biological significance. Virchows Arch. 2000;437:581-90.

7. Elhanafi S, Saadi M, Lou W, Mallawaarachchi I, Dwivedi A, Zuckerman M, et al. Gastric polyps: Association with Helicobacter pylori status and the pathology of the surrounding mucosa, a cross sectional study. World J Gastrointest Endosc. 2015;7:995-1002.

8. Dirschmid K, Platz-Baudin C, Stolte M. Why is the hyperplastic polyp a marker for the precancerous condition of the gastric mucosa? Virchows Arch. 2006;448:80-4.

9. Jalving M, Koornstra JJ, Wesseling J, Boezen HM, de Jong S, Kleibeuker JH. Increased risk of fundic gland polyps during long term proton pump inhibitor therapy. Aliment Pharmacol Ther. 2006;24:1341-8.

10. Bianchi LK, Burke CA, Bennett AE, Lopez R, Hasson H, Church JM. Fundic gland polyp dysplasia is common in familial adeno-matous polyposis. Clin Gastroenterol Hepatol. 2008;6:180-6.

11. Hofgartner WT, Thorp M, Ramus MW, Delorefice G, Chey WY, Ryan CK, et al. Gastric adenocarcinoma associated with fundic gland polyps in a patient with attenuated familial adenomatous polyposis. Am J Gastroenterol. 1999;94:2275-81.

12. De Vries AC, van Grieken NC, Looman CW, Casparie MK, de Vries E, Meijer GA, et al. Gastric cancer risk in patients with premalignant gastric lesions: A nationwide cohort study in the Netherlands. Gastroenterology 2008;134:945-52.

13. Tomasulo J. Gastric polyps. Histologic types and their relations- hip to gastric carcinoma. Cancer. 1971;27:1346-55.

14. Grantcharov TP, Kehlet H. Laparoscopic gastric surgery in an enhanced recovery programme. Br J Surg. 2010;97:1547-51.

15. Kehlet H, Wilmore DW. Evidence-based surgical care and the evolution of fasttrack surgery. Ann Surg. 2008;248:189-98.

16. Wang HL, Fang F, Lu CM, Wang DR, Li P, Fu P. Safety of fast-track rehabilitation after gastrointestinal surgery: Systematic review and meta-analysis. World J Gastroenterol. 2014;20:15423-39.

17. Haverkamp L, Weijs TJ, van der Sluis PC, van der Tweel I, Ruurda JP, van Hillegersberg R. Laparoscopic total gastrectomy versus open total gastrectomy for cancer: A systematic review and meta-analysis. Surg Endosc. 2013;27:1509-20. 